realised that it was associated with a variable radio source (see for example Clark \& Murdin, Nature 276, 44; 1978; Ryle et al. Nature 276, 571; 1978). An $\mathrm{X}$-ray source A1909+04 is also in the right position and it is located in line with the supernova remnant W50. Margon and his colleagues are the first to observe the unusual optical emission features. Two in particular lie either side of $H \alpha$, the infrared one of which moved to longer wavelengths by $150 \AA$, the red feature moving in the opposite sense by $70 \AA$, in just four nights observing. SS433 presents a challenge; perhaps it too will become a regular at the Texas conferences.

\section{Pathogenic mechanisms of mycoplasmas}

\author{
from D. B. Archer
}

Mycoplasmas are a diverse group of wall-less prokaryotes. They have been implicated as possible causal agents in a multitude of diseases in animals, plants and insects but have been demonstrated to be causative in relatively few instances and in only a handful of cases is there any information which bears directly on the pathogenic mechanisms involved. However, from the Proceedings of the 2nd Conference of the International Organisation for Mycoplasmology (IOM) (Zentralbl. Bakt. Parasitnek. Infekt. Hyg. 241; 1978) it is clear that an understanding of the pathogenic mechanisms is now being worked towards.

The diversity in form of mycoplasmas reflects the range of diseases and mechanisms involved. Some mycoplasmas are motile and motility may be necessary for these organisms to reach their target site, although a nonmotile variant of Spiroplasma citri is known to be pathogenic when injected into host phloem cells by an insect vector (Townsend et al. J. gen. Microbiol. 100, 15; 1977). Mycoplasmas lack flagella and their mechanisms of motility are poorly understood although an actin-like protein has been reported in Mycoplasma pneumoniae (Neimark Proc. natn. Acad. Sci. U.S.A. 74, 4041; 1977). However, Rodwell et al. (IOM Proceedings) showed

D. B. Archer is in the CSIRO Division of Animal Health, Animal Health Laboratory, Parkville, Australia. that no mycoplasma protein coelectrophoresed with rabbit muscle actin by two-dimensional gel electrophoresis. Although Rodwell et al. could not detect a mycoplasma protein having a high affinity for deoxyribonuclease 1, a characteristic of eukaryotic actin, Kahane et al. (IOM Proceedings) were able to isolate a multiprotein complex having a high affinity for muscle myosin.

Attachment to host cells is of primary importance in the pathogenicity of Mycoplasma gallisepticum and M.pneumoniae, among others. A M.pneumoniae membrane protein (P1) is required for the attachment to host tracheal cells (Hu et al. J. exp. Med. $145,1328 ; 1977)$ and although these workers could not detect any staining of M.pneumoniae proteins with PAS, a carbohydrate stain, it is of interest to know whether P1 is identical to the recently isolated glycoprotein from M.pneumoniae (Kahane \& Brunner Infect. Immun. 18, 273; 1977) because carbohydrate has been implicated in the attachment of this organism to host epithelium (Powell et al. Infect. Immun. 13, 959; 1976). Apart from the presence of a glycoprotein in M.pneumoniae, and possibly M.gallisepticum (Goel \& Lemcke Ann. Microbiol. 126, 299; 1975) there are no confirmed reports of glycoproteins in any other mycoplasmas. If carbohydrate is important in the host cell attachment of other mycoplasmas it need not be through a glycoprotein and Huang (Nature 276, 624; 1978) has recently pointed out that glycolipids can be involved in cell adhesion, although glycolipids do not seem to be involved in the host cell adhesion of $M$. pneumoniae (Hu et al. op. cit). Both $M$. pneumoniae and $M$. gallisepticum adhere to erythrocytes and this attachment has been used as a convenient model for natural host cell attachment. It seems that glycophorin is the major erythrocyte receptor for $M$. gallisepticum (Banai et al. IOM Proceedings; Infect. Immun. 21, 365; 1978) whereas this is not the case with $M$. pneumoniae (Feldner et al. IOM Proceedings), although bindings of $M$. pneumoniae to host epithelial cells is reduced by pretreating host cells with neuraminidase (Powell et al. Infect. Immun. 13, 959; 1976: Gabridge, IOM Proceedings). These mycoplasmas also bind to glass and plastic so it is of interest to know what determines specificity of binding in vivo. Sialic acid is of importance in the binding of M.gallisepticum to erythrocytes but this residue alone probably could not provide specificity of host. Isolation of the presumed binding proteins of mycoplasmas and determination of their affinities for target sites would enable some judge- ments to be made on the specificity of mycoplasma-host cell interactions.

Host cell attachment may not be as important in determining specificity as the production of a mycoplasma toxic substance or susceptibility of the host to it. Indeed, there is no evidence for high affinity host cell attachment by most mycoplasmas. Unfortunately, information on toxic products of mycoplasmas is meagre. Hydrogen peroxide and ammonia are produced by some mycoplasmas and may be toxic factors, particularly if the association of the mycoplasma with the host is intimate (for example $M$. pneumoniae) or if the amount of hydrogen peroxide is large (Mycoplasma mycoides subsp. capri). Specificity may more easily be explained by the production of a toxic compound which has selectivity of action, but little information on such substances is available. Characterised toxic cell products are the galactan of M.mycoides subsp. mycoides (Lloyd et al. J. med. Microbiol. 4, 425; 1971: Buttery et al. J. med. Microbiol. 9, $379 ; 1976)$ and the exotoxin of $M y c o-$ plasma neurolyticum (Tully, INSERM 33, 317; 1974). Also, low molecular weight toxins have been isolated from S.citri (Daniels \& Meddins INSERM 33, 195; 1974: Daniels IOM Proceedings). One hopes that purification and characterisation of such toxic products will lead to experiments designed to determine their modes of action. Exotoxins were not detected, however, in culture filtrates of $M$. gallisepticum, an organism known to be neurotoxic. (Thomas Ann. N.Y. Acad Sci. 143, 218; 1967). Some mycoplasmas bind to and transform lymphocytes (Ginsburg \& Nicolet Nature new Biol. 246, 143; 1973; Cole et al. IOM Proceedings; Wise et al. IOM Proceedings) and form caps (Stanbridge IOM Proceedings; Stanbridge \& Weiss Nature 276, 583; 1978) demonstrating mitogenic activity in vitro. Also, Cole et al. (IOM Proceedings) reported that mycoplasmas induce the release of cytotoxic factors from lymphocytes. The in vivo importance of mycoplasma interactions with lymphocytes is unknown but provides a plausible explanation for many of the effects of mycoplasma infection.

In conclusion, although information on the pathogenic mechanisms of mycoplasmas is still limited the situation is improving. Specificity remains a major problem which can only be overcome by characterisation of the cell residues involved in host cell attachment of those mycoplasmas which do attach, and by isolation and characterisation of toxic factors. Demonstration of in vivo mitogenicity would clearly be a major advance in our understanding of the pathogenic mechanisms of mycoplasmas. 